\title{
Fourier-imaging of single self-assembled CdSe nanoplatelet chains and clusters reveals out-of-plane dipole contribution
}

\author{
Jiawen Liu ${ }^{1}$, Lilian Guillemeney ${ }^{2}$, Arnaud Chouxx ${ }^{1}$, Agnès Maître ${ }^{1}$, Benjamin Abécassis ${ }^{2}$ and \\ Laurent Coolen ${ }^{1}$
}

\begin{abstract}
${ }^{1}$ Sorbonne Université, CNRS, Institut de NanoSciences de Paris, INSP, F-75005 Paris, France
${ }^{2}$ Univ Lyon, CNRS, École Normale Supérieure de Lyon, Laboratoire de Chimie UMR 518246 allée d'Italie, F-69007 Lyon, France
\end{abstract}

\section{Supporting Information}

\section{A - Precision of the $\eta$ coefficients}

We discuss here the precision of the fits of the radiation pattern for various examples of emitters under different measurement configurations. Arrows point to the pattern features which are used primarily to assess the fit relevance : the main ones are the side lobes and dips, keeping in mind that the $\theta \sim 0$ values are more prone to noise fluctuations because they originate from only a few camera pixels, and that the highest angles $\left(\theta>60^{\circ}\right)$ seem affected by geometric aberrations ${ }^{2}$. For all three cases (figs. S1, S2(a), S3), we estimate the uncertainty to 0.03 for the out-of-plane dipole and 0.05 for the minor in-plane dipole. Note that the achieved precision is better for the out-of-plane dipole because the measurement configurations (immersion 1.5 N.A. objective, 130-nm PMMA layer for fig. S1 and no PMMA for figs S2(a) and S3) were chosen in order to optimize specifically the measurement of this coefficient. The index of PMMA is close to 1.5 so that there is no optical effect of the PMMA/glass interface. We also compare in fig. S2(b) the radiation patterns of single NPLs measured either with or without a 30-nm PMMA layer. The two configurations are qualitatively different : in the latter, the collected emission is transmitted through the air-glass interface, while in the former it is a sum of the direct radiation and the radiation reflected by the air-PMMA interface. However, in both configurations we obtain very close coefficients.

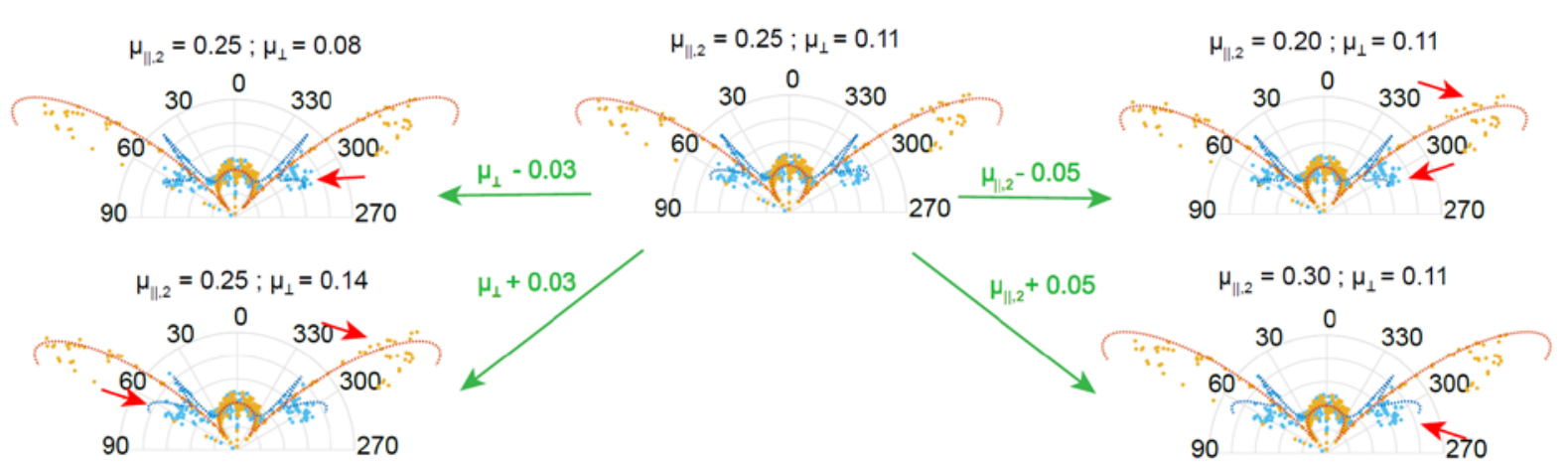

Figure S1: Radiation pattern of a long NPL chain covered by a 130-nm PMMA layer, fitted with different $\eta$ factors. 
(a)

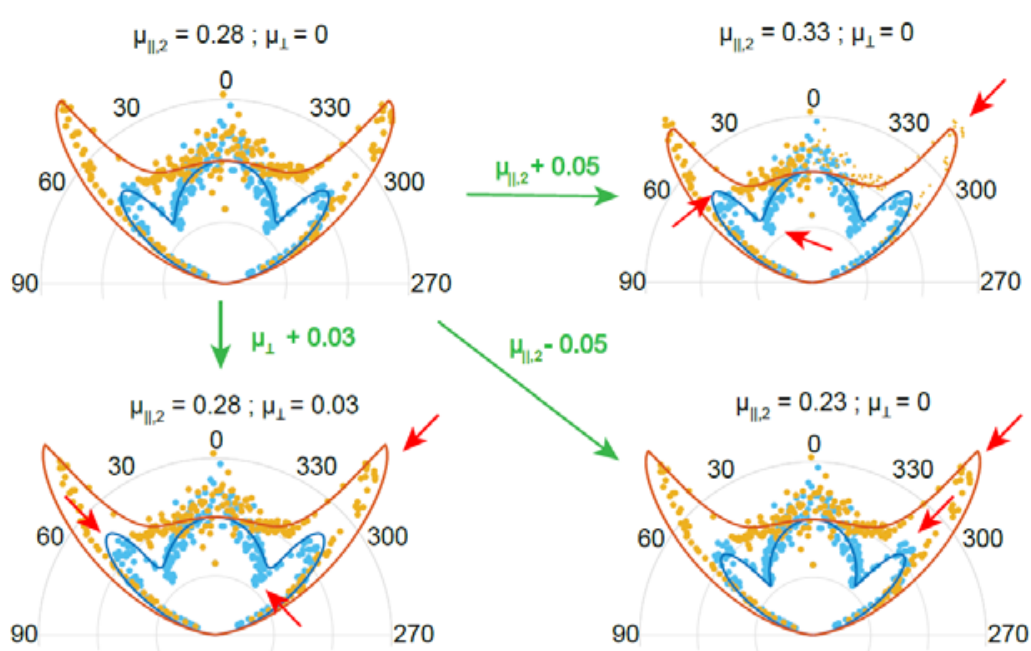

(b)

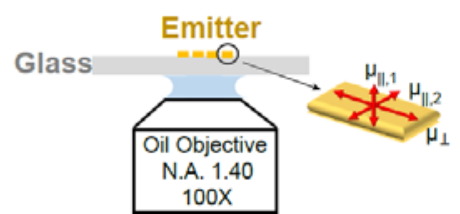

$$
\mu_{\|, 2}=0.28 ; \mu_{\perp}=0
$$
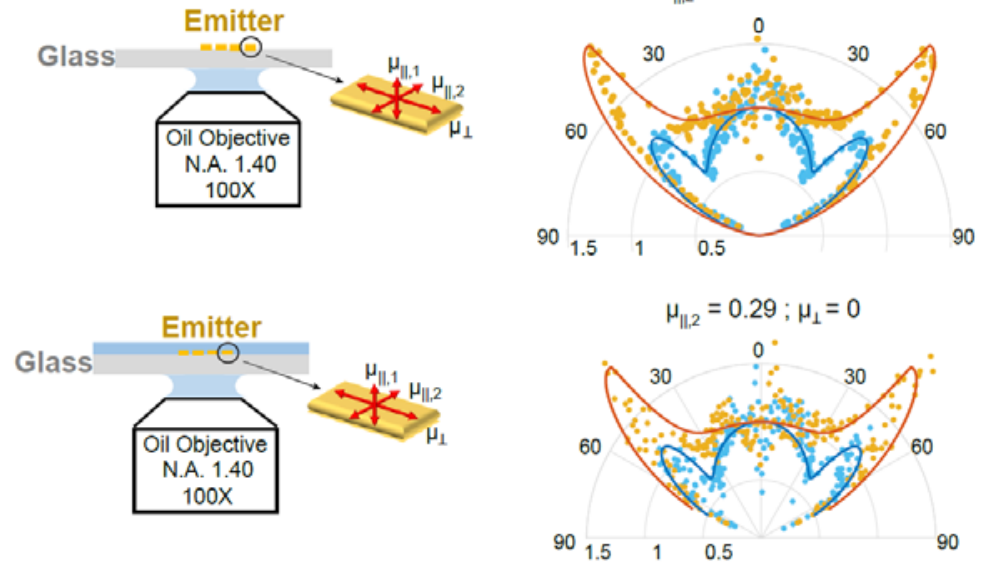

Figure S2 : (a) Radiation pattern of a single NPL (with no PMMA layer), fitted with different $\eta$ factors. (b) Radiation patterns of 2 typical single NPLs measured under different conditions (with / without 30nm PMMA layer).

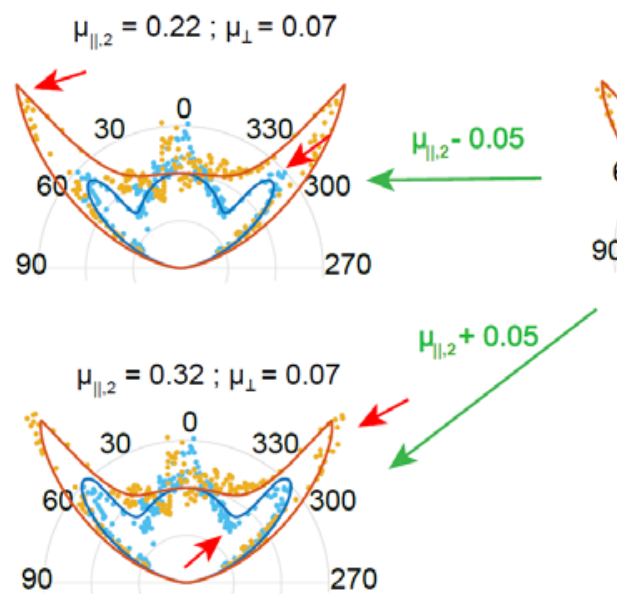

$$
\mu_{\| .2}=0.27 ; \mu_{\perp}=0.07
$$$$
\mu_{\| \mid .2}=0.27 ; \mu_{\perp}=0.04
$$
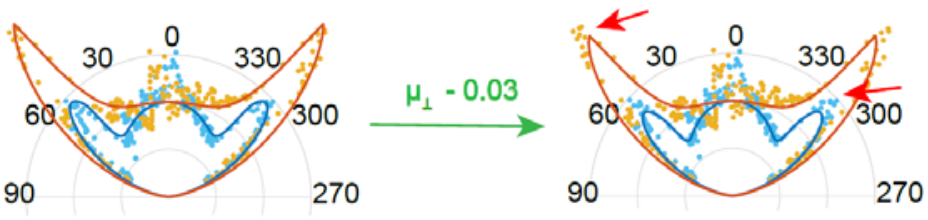

90 270
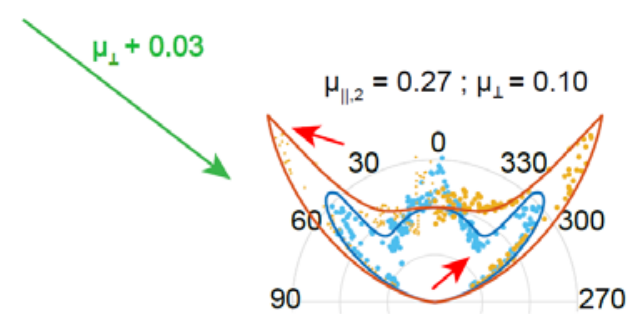

Figure S3 : Radiation pattern of a NPL cluster (with no PMMA layer), fitted with different $\eta$ factors. 


\section{B - Identification of single NPL clusters}

As explained in the paper, we deposited isolated (non assembled) NPLs on a glass slide and detected point-like emitters. We used photon post-selection (fig. S4) to eliminate the contributions both from multiexcitonic recombination and substrate self-luminescence, as these contributions correspond to short (a few ns) decay : we plotted the autocorrelation $g^{(2)}(\tau)$ function with only the photons detected more than 2 ns after the excitation laser pulse, originating from excitonic decay.
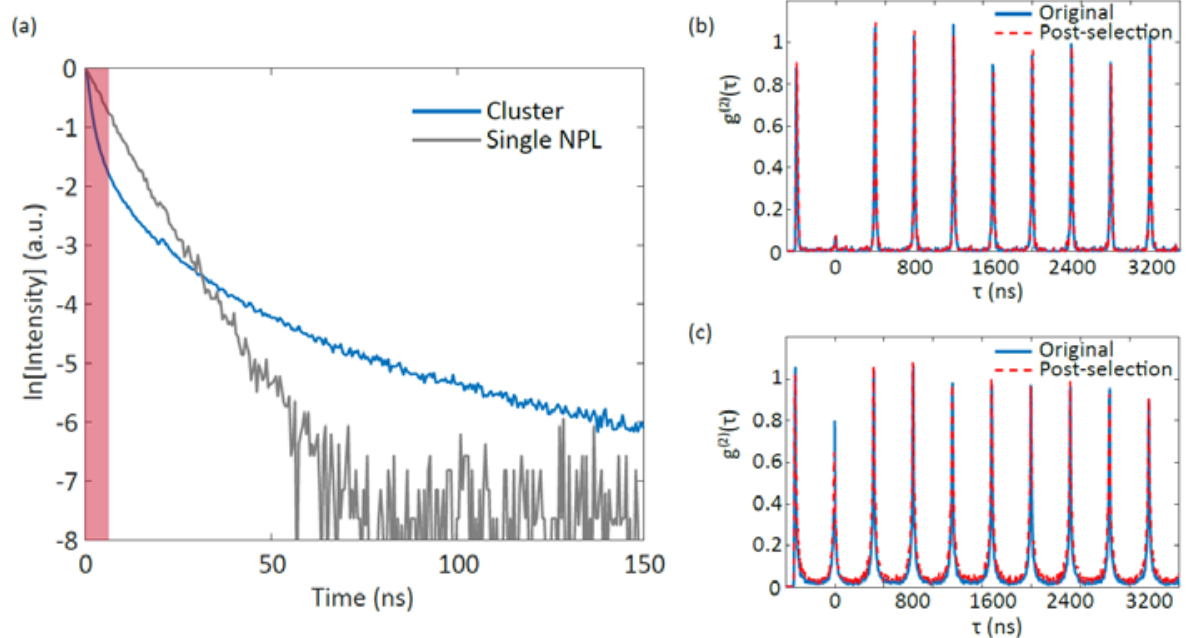

Figure S4 : (a) Decay curves from two emitters, one attributed to a single NPL and the other to a cluster. Photon correlation curves of (b) the single NPL and (c) the cluster : as compared to the original curve (full line), the post-selected curve (dotted line) was obtained by selecting only photons after 2-ns in the decay curve (excluding the red interval in fig. $54(a)$ ). The post-selected curve improves partially the antibunching for the cluster, however not to a full single-photon emission.

We then extracted the zero-delay peak weight $g_{0}$ defined as the integrated area of the zero-delay peak divided by the average of the areas of the other peaks. The number of emitters was estimated as $N=1 /\left(1-g_{0}\right)$ and emitters with $g_{0} \leq 0.1$ can be considered as single platelets, while the others were assumed to be clusters. We present here additional characterizations showing that the emitters identified as clusters present behaviours qualitatively different from the single platelets and closer to the self-assembled chains:

- the emitters all show intensity fluctuations (fig. S5(a)), however the single platelets usually present clear binary on-off switching typical of blinking, while the clusters present a more chaotic intensity-time trace as might be expected from a sum of a few independently-blinking emitters. For the NPL chains, the intensity fluctuations are very low, which can be attributed to an averaging of the blinking of many emitters. If we quantify roughly the amount of blinking by the blinking factor (standard deviation) : $S=\sqrt{\frac{1}{N-1} \sum_{i=1}^{N}\left|I_{i}-\bar{I}\right|^{2}}$, it appears that the blinking factor is generally lower for the clusters than for the single platelets (fig. S5(d)). Finally the blinking factor for the chains (not plotted here) was typically 0.05-0.1. 
- the decay curve for the single platelets is an exponential with an average decay time of 12 ns. This is a typical behaviour for exciton recombination in a semiconductor nano-emitter. For the clusters and the chains, on the other hand, for any emission intensity the decay is much faster and highly non-exponential. If we consider the time for the decay curve to decrease by a factor 10 (fig. S5(c)), it ranges between 4 and 17 ns for the single platelets while it is only 1.5-3.5 ns for the clusters. This type of behaviour for self-assembled NPLs was reported already in refs. 3-6 and attributed to FRET-mediated quenching : excitons are transferred by FRET to some extremely fast quenching defect platelets.
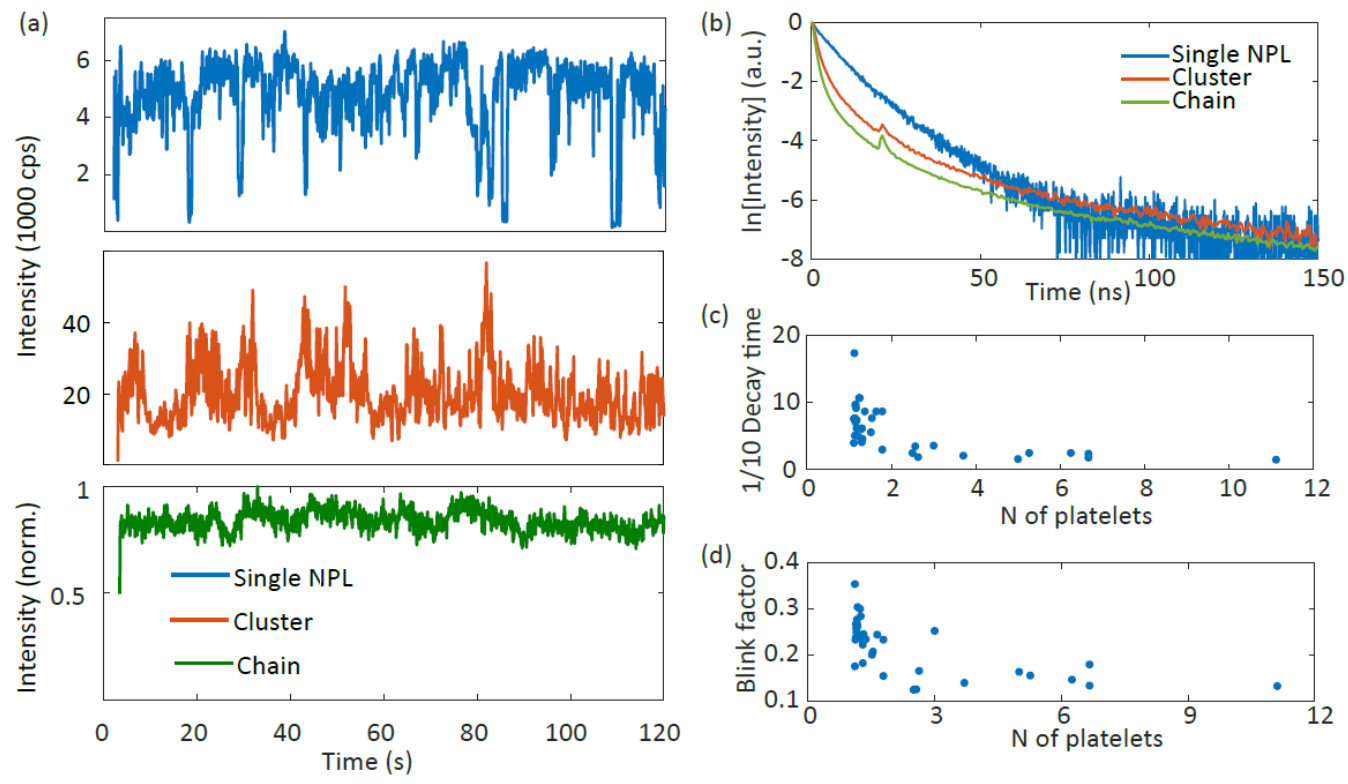

Figure S5: (a) Intensity-time trace of typical single platelet (top), NPL cluster (middle) and long NPL chain (bottom). (b) Decay curves of these emitters (the small peak at 20 ns is related to electronic reflections of the counting system). (c) Plot of the decay time (defined as the time of decay by a factor 10) as a function of the number $N$ of platelets of the emitter. (d) Blinking factor (defined as the intensity standard deviation) as a function of the number $N$ of platelets of the emitter.

\section{C - FDTD simulation of dielectric antenna effect}

We used FDTD simulations to examine how the shape of the dielectric structure affects its own radiation. We built up models with different structures as shown in fig. S6(a) : a symmetric $10 \times 10 \times 10 \mathrm{~nm}^{3}$ cube (left), a single CdSe NPL (middle) and a stack of $201 \mathrm{NPLS}$ (right) with a 5.7-nm center-to-center distance. The size of a single NPL was $20 \times 7 \times 1.5 \mathrm{~nm}^{3}$, as in our experiments. The length of the chain was $1500 \mathrm{~nm}$. Here we set the refractive index of all the dielectric structures as $2.64+0.44 i$, which is the refractive index of bulk zinc-blende $\mathrm{CdSe}$ at $550 \mathrm{~nm}$ wavelength ${ }^{1}$. The platelets are set on a glass (index 1.5) substrate and covered by a 130-nm PMMA layer (index 1.5). We positioned a monitor $30 \mathrm{~nm}$ below the structure with a large enough lateral expansion $\left(15 \times 15 \mu^{2}\right)$ to properly collect all the near field electromagnetic waves. We then numerically integrated the projected electrical field intensity $\mathrm{E}^{2}$ in the far field. We defined $I_{x}, I_{y}, I_{z}$ as the intensities detected respectively from a dipole 
along the $x, y$ or $z$ axis. We checked that, when the structure is a cube, we obtain $I_{x}=I_{y}=$ $I_{z}$ as expected for an isotropic structure. We show below (in relative units) the values calculated for the single NPL and the NLP chain. In order to analyze the effect of the separation between the stacked NPLs, we also consider the case of a continuous CdSe block in dimensions of $20 \times 7 \times 1200 \mathrm{~nm}^{3}$ (fig. S6(a)).

\begin{tabular}{|l|c|c|c|}
\hline & $I_{x}$ & $I_{y}$ & $I_{z}$ \\
\hline Single NPL & 7 & 53 & 42 \\
\hline NPL chain & 9 & 46 & 32 \\
\hline CdSe block & 59 & 28 & 10 \\
\hline
\end{tabular}

Table S1 : emission intensities of dipoles along $x, y$ or $z$ directions in three different configurations.

For the single NPL, as expected in an elongated dielectric antenna, the emission is much stronger for an in-plane dipole ( $y$ or $z$ ) than for an out-of-plane dipole $(x)$, and it is stronger for the main axis of the platelet $(y)$ than for its shorter axis (z).

This remains true for the NPL chain : the main effect is the effect of each single NPL which enhances the in-plane dipoles ( $y$ and $z$ ) with respect to the out-of-plane dipole $(x)$. However, the ratio of $x$ with $y$ and $z$ is slightly larger for the chain: there is a slight dielectric antenna effect of the stacking axis $x$ of the chain. But this effect only weakly restores the $x-$ dipole contribution which was quenched by the NPL flat shape. This is very different from the case of an elongated CdSe block, where the x-axis dipole is strongly enhanced by dielectric antenna effect as compared to the other axes.

We plot in fig. S6(b) the evolution of the antenna effect as a function of the number of platelets (normalized to the case of a single platelet). As compared to the single platelet case, the enhancement of the $x$ dipole with respect to the $y$ and $z$ dipoles by the antenna effect of the whole NPL chain is only by $26 \%$. We observe that this antenna effect begins to appear with around 2-10 platelets, corresponding to a 10-60 nm length : this is when the length of the chain begins to be larger than the width of the platelets. When more than 15 NPLs are stacked, the same results are found for any number of platelet so that the knowledge of the exact chain length is not important. 
(a)

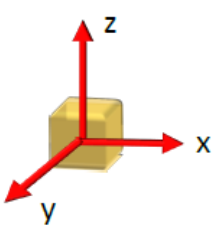

(b)

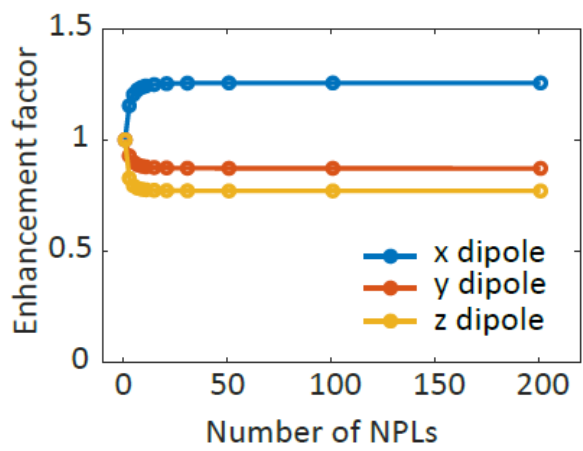

(d)
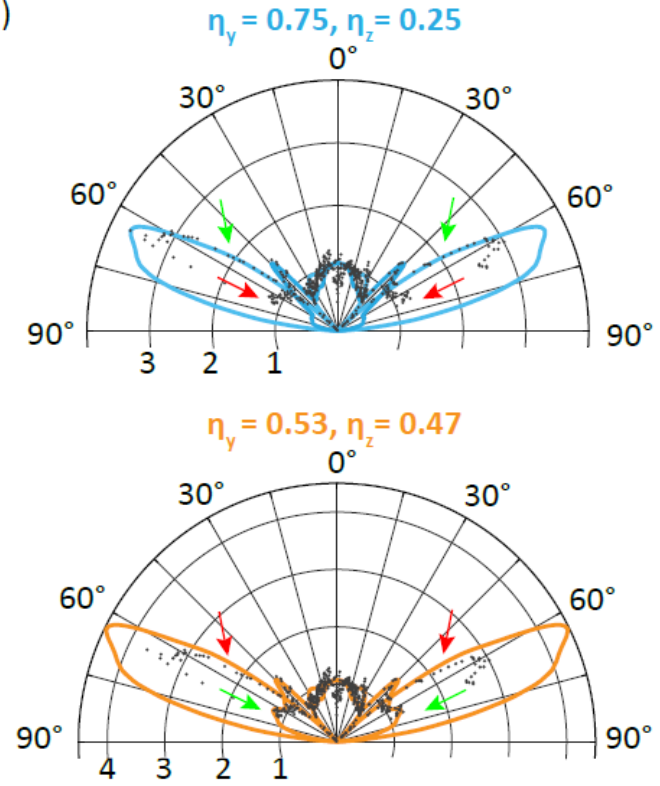
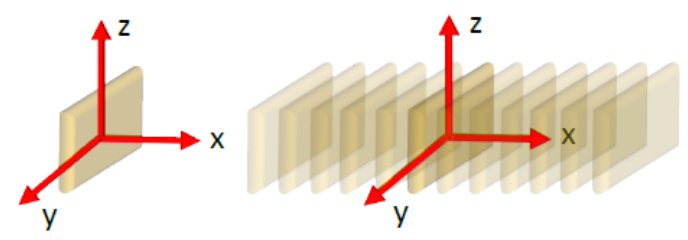

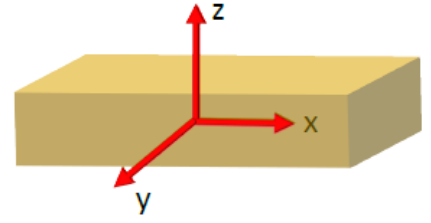

(c)
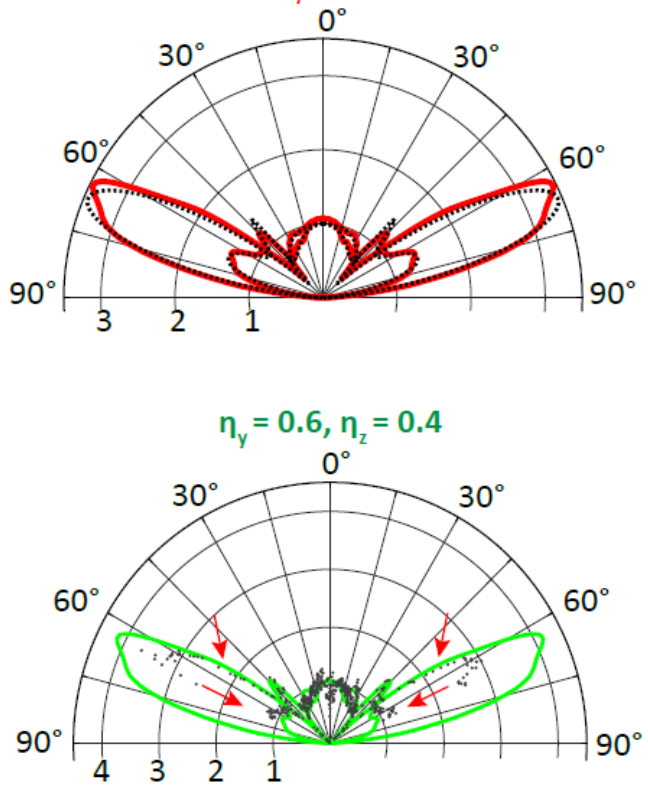

$\eta_{y}=0.5, \eta_{z}=0.5$

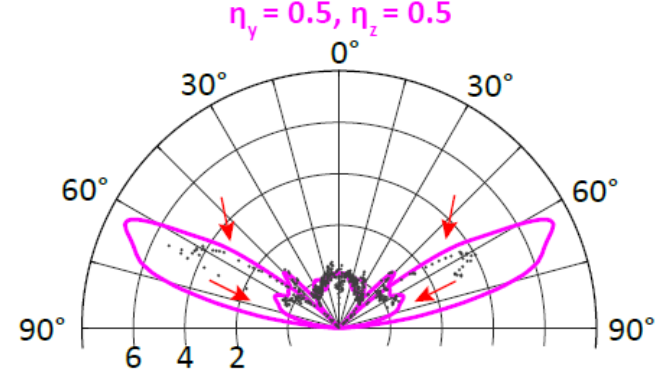

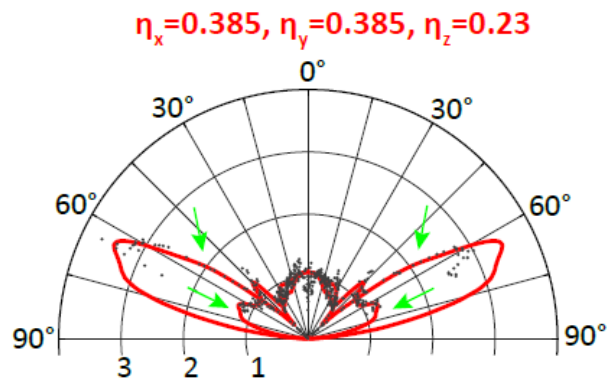

Figure S6: (a) Different models of (from left to right) a symmetric $10 \times 10 \times 10 \mathrm{~nm}^{3}$ cube, a single $20 \times 7 \times 1.5$ $\mathrm{nm}^{3} \mathrm{NPL}$, a stacked NPLs chain and a $20 \times 7 \times 1200 \mathrm{~nm}^{3} \mathrm{CdSe}$ block. (b) Evolution of $I_{x}, I_{y}, I_{z}$ as a function of the number of platelets in the chain (normalized to the case of a single NPL). (c) Radiation patterns of the dipoles $\eta_{x}=0.12, \eta_{y}=0.62, \eta_{z}=0.26$ placed in a homogeneous medium (black; analytical calculation) and $\eta_{x}=0.385, \eta_{y}=0.385, \eta_{z}=0.23$ placed inside a NPL chain (red; numerical model). (d) Comparison of the experimental radiation pattern of a NPL chain with the theoretical radiation 
patterns of several dipole components inserted inside a NPL chain, the first 4 curves being with no outof-plane dipole, and the $5^{\text {th }}$ curve with 3 dipoles providing the best fit. The green and red arrows show respectively where the agreement and disagreement between experiment and theory are the most noteworthy (keeping in mind that the highest angles may be affected by aberrations).

These calculations mean that, if a sum of 3 incoherent dipoles of equal moments $\left(\eta_{x}=\right.$ $\eta_{y}=\eta_{z}$; we recall that the $\eta$ coefficients are proportional to the square of the dipole moments, so they are proportional to the emitted intensity) is inserted in a platelet inside a chain, the $y$ dipole will be enhanced $46 / 9=5.1$ times with respect to the $x$ dipole, and the $z$ dipole 3.55 times. A dipole with $\eta_{x}=0.33, \eta_{y}=0.33, \eta_{z}=0.33$ inserted in a NPL chain should thus behave like a dipole with $\eta_{x}=0.10, \eta_{y}=0.53, \eta_{z}=0.37$ inside a homogeneous medium. Again, we see that the $y$ and $z$ dipoles are enhanced with respect to the $x$ dipole.

In order to account for the experimental observation that the NPL chain behaves as a sum of dipole contributions $\eta_{x}=0.12, \eta_{y}=0.62, \eta_{z}=0.26$, we thus need to place a sum of dipoles $\eta_{x}=0.385, \eta_{y}=0.385, \eta_{z}=0.23$ inside the NPL chain, so that the antenna effect modifies the latter dipole sum into the former. We can verify that such a dipole would indeed match with our measurements by plotting in fig. S6(c) the radiation patterns of the former dipole in a homogeneous medium and the latter dipole inside a NPL chain : the two are in excellent agreement.

Eventually, we calculate that a sum $\eta_{x}=0.385, \eta_{y}=0.385, \eta_{z}=0.23$ placed inside a single isolated platelet would be equivalent to a sum $\eta_{x}=0.08, \eta_{y}=0.63, \eta_{z}=0.29$ inside a homogeneous medium. In conclusion, in order to explain the experimental observation of a $\mathbf{0 . 1 2}$ out-of-plane dipole component for the NPL chain radiation purely by a dielectric antenna effect, we would need to have a $\mathbf{0 . 0 8}$ out-of-plane dipole in a single NPL.

As additional verification, we plot in fig. S6(d) the experimental radiation pattern of a NPL chain and compare it with the simulated radiation patterns of a NPL chain. In the first 4 cases, we insert various dipoles with no out-of-plane component and none of these theoretical cases is able to describe the experimental data: when $\eta_{z}=0.25$ (blue curve), the bigger lobes (along $\Phi \sim 65^{\circ}$ ) are reasonably fit but not the small lobes (as indicated by red arrows). As the value of $\eta_{z}$ increases, the big and small lobes will enlarge simultaneously. One can fit the small lobes well when $\eta_{z}=0.47$ (yellow curve), but at the same time the fit to the big lobes is lost. However, in the $5^{\text {th }}$ case, we put the calculated dipoles $\eta_{x}=0.385, \eta_{y}=0.385, \eta_{z}=0.23$ into the same simulation and a good fit is obtained. This shows that the dielectric antenna alone is not able to generate the observed out-of-plane dipole in the NPL chain, if a significant out-of-plane component was not already present in the single NPL.

\section{D - Influence of excitation power}

We have estimated in ref. 7 (see S.I. - section E for discussions of this value) that under $5-\mathrm{nW}$ pulsed excitation an isolated platelet is excited with $8.10^{-3}$ probability for each pulse, so that we are well within the linear regime of the platelets. Following this value, a $320-\mathrm{nm}$ diameter laser spot on a NPL chain should excite around 55 platelets so that 0.44 exciton should be excited per pulse in the entire chain (possibly less because the chains have vertical 
orientation so their absorption cross section should be lower). We thus expect to have few exciton-exciton interaction because less than one exciton is created per laser pulse for all emitters considered here : single platelets, clusters, short and long chains.

Figure S7 plots the emitted intensity and the decay curve for the on-states of a single NPL as a function of the excitation power. For the range of powers used here (5-10 nW), the decay curve is independent on the excitation power, and the photon count follows a linear dependence on the excitation power, confirming that we are indeed in the linear regime of the nanoplatelets.
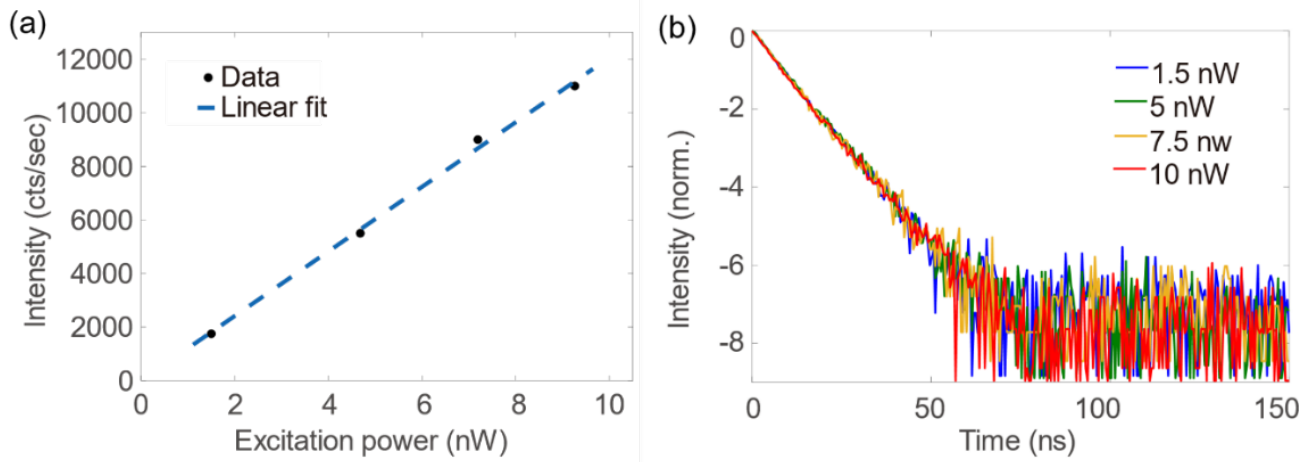

Figure S7: Power-dependent intensity curve (a) and decay curves (b) of the on-states of a same single nanoplatelet (NPL) emitter under different excitation powers.

As a supplementary characterization, we show on fig. S8 the blinking (a) and antibunching (b) curves of a single NPL at three different excitation powers : while blinking becomes more frequent as the power is increased, suggesting a contribution to blinking from a photo-induced mechanism, the antibunching curve remains the same.
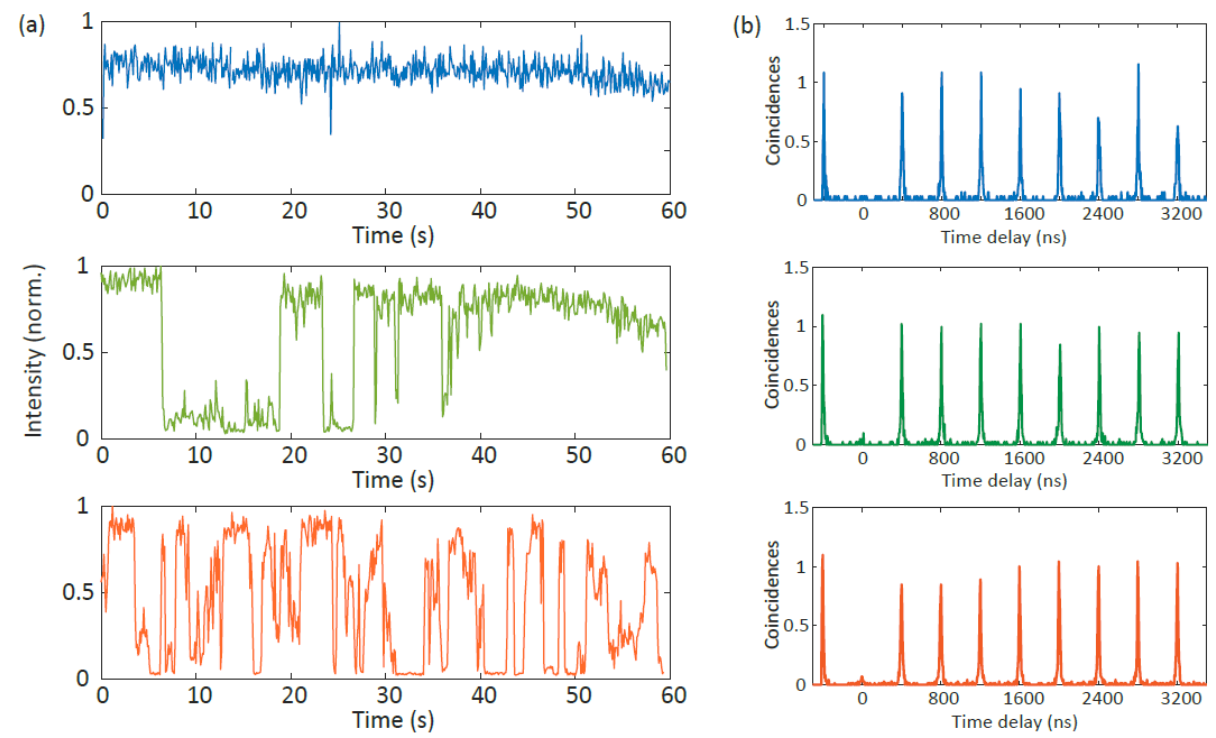

Figure S8: (a) Intensity-time trace and (b) photon correlation function (normalized) of the same single nanoplatelet under (top) $1.5 \mathrm{nW}$, (middle) $5 \mathrm{nW}$ and (bottom) $10 \mathrm{nW}$ excitation. 
We also plot on fig. S9 the decay curves and polarization analysis of a NPL chain under different excitation powers. The decay curve remains the same up to around $10 \mathrm{nW}$ and becomes faster at higher powers, possibly because the quenchers responsible for the fast decay become more present at higher excitation power. The degree of polarization is exactly the same $(\delta=0.49 \pm 0.01$ ) for any value of polarization. This value is consistent with our values from fitting Fourier planes : $\eta_{\|, 2}=0.25, \eta_{\perp}=0.11$. This indicates that the out-of-plane dipole remains present for all excitation powers : if it disappeared completely (while keeping $\eta_{\|, 1} / \eta_{\|, 2}$ constant), the degree of polarization would drop to only 0.27 . If the out-of-plane component decreased only slightly to 0.08 , the degree of polarization would change to 0.53 , which would still be measurable. Eventually we can exclude that the measured out-of-plane dipole component originates from multi-excitonic effect.

(a)
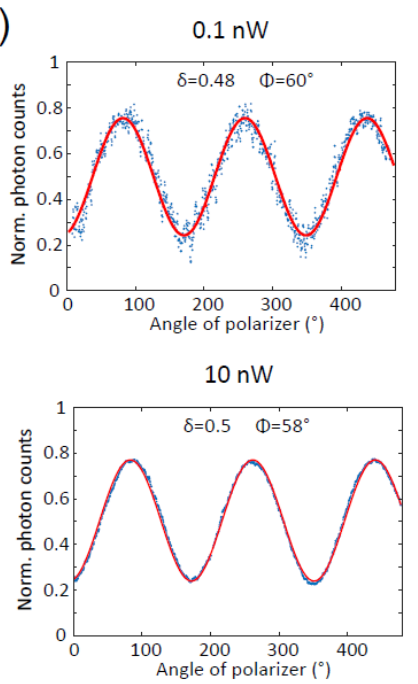
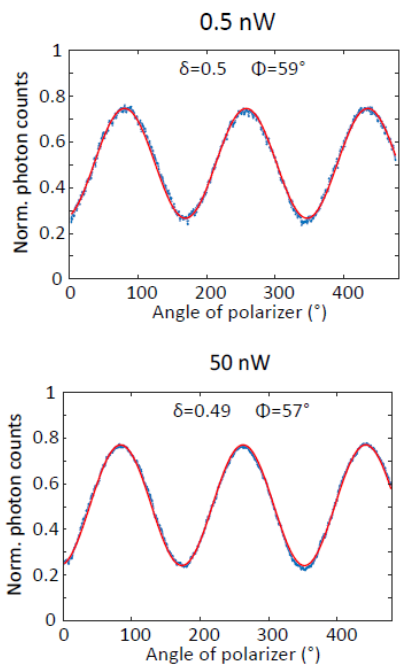

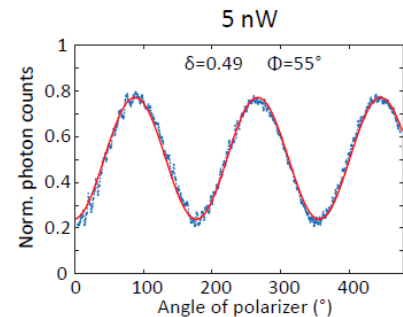

(b)

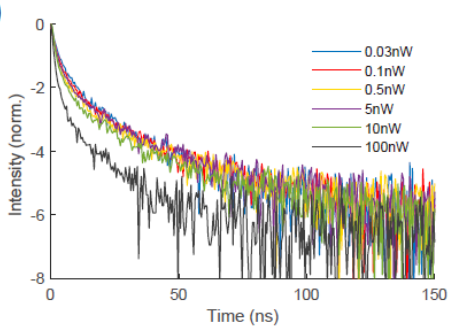

Figure S9 : (a) Polarization analysis curves and fitted $\delta$ and $\Phi$ parameters and (b) Decay curves of a given NPL chain under different excitation powers.

\section{E - Polarization effect on decay curves and spectra}

The decay curve (fig. S10, left) and the emission spectrum (fig. S10, right) of a NPL chain showed no effect of adding a polarizer : both polarizations parallel and perpendicular to the chain axis showed the same properties. This indicates that the different dipole contributions (i) do not originate from different emitter populations with different dynamics and (ii) do not originate from states of different energies (within the room-temperature line width). 

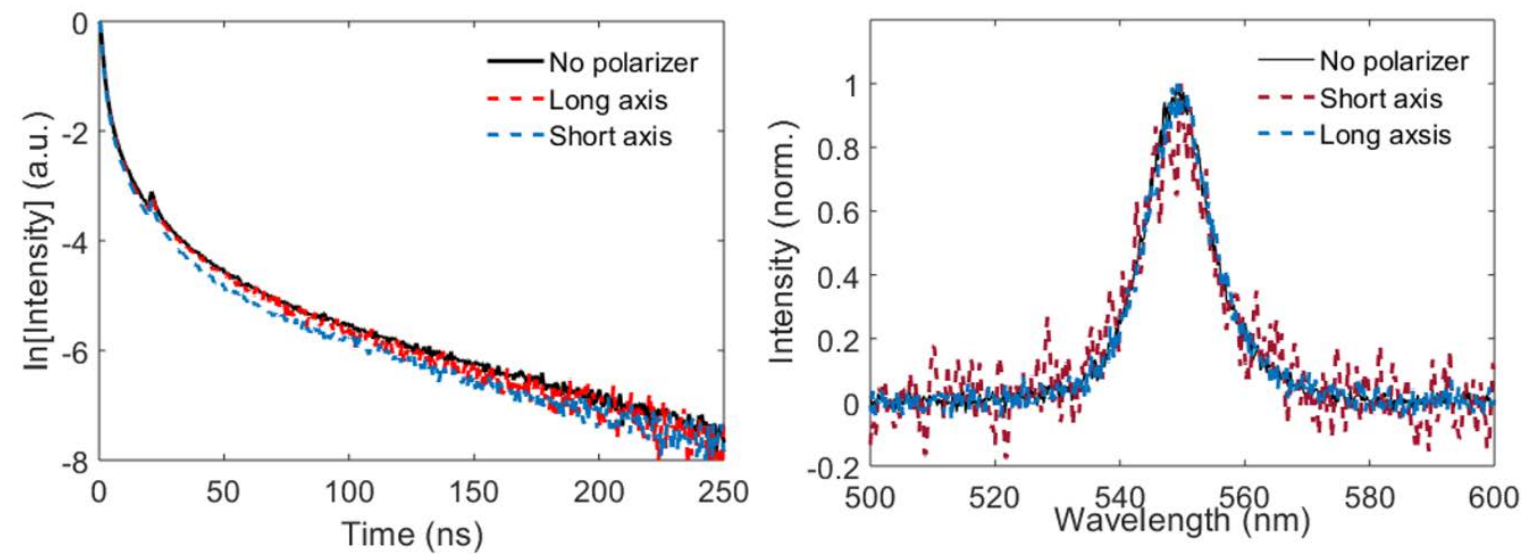

Figure S10 : decay curve and emission spectrum of a NPL chain analysed with a polarizer either parallel or perpendicular to the chain's long axis.

\section{F - Temperature spectral dependence}

Figure S10(a) shows the emission spectra of the same NPL chain at different temperatures. The measurement was performed with an Oxford HiRes II cryostat, the 150- $\mu \mathrm{m}$ glass sample was attached by a silver lacquer to a copper holder (note that the temperature value is the one given by the controller located on the other side of the copper holder : due to imperfect substrate thermal conduction, the actual temperature at the emitter's position might be 5-10 K higher). The 5-K and 50-K spectra show clearly the second redshifted peak which was reported and debated by several groups. This peak is dominant below $100 \mathrm{~K}$ and decreases after $150 \mathrm{~K}$. Above $200 \mathrm{~K}$, only the excitonic emission peak remains (the excitonic peak shifts as a function of temperature due to a change in the bulk band gap energy). We can thus exclude any contribution from the second peak at room temperature.
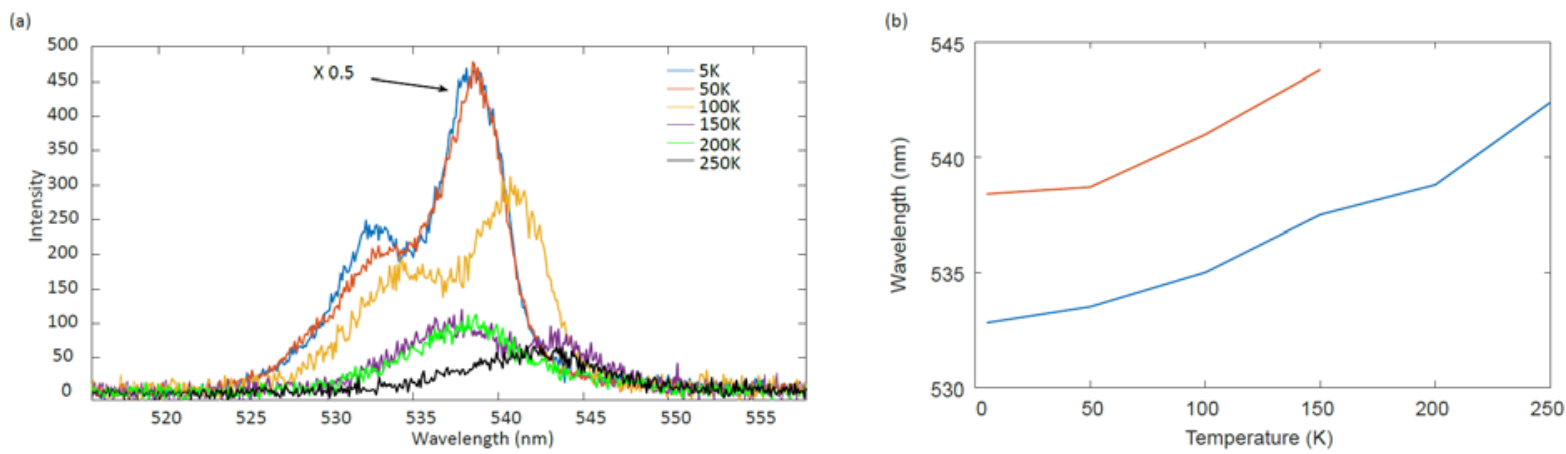

Figure S11 : (a) NPL chain emission spectrum at various temperatures. (b) Peaks positions as a function of the temperature.

\section{G - Tilt of the NPL stacks}

We analyse here a TEM image of NPL chains (fig. S12(a)) in order to evaluate the angle by which the NPLs may be tilted with respect to the stacking axis due to the slight stacking 
disorder. We can define tilt angles $\beta_{1}$ and $\beta_{2}$ (fig. S12(b)) : while the out-of-plane tilt angle $\beta_{2}$ cannot be evaluated on the TEM images, we can measure the in-plane angle $\beta_{1}$ for each NPL of a given NPL thread. The resolution on this angle is estimated to $3^{\circ}$ (fig. S12(c)).

(a)

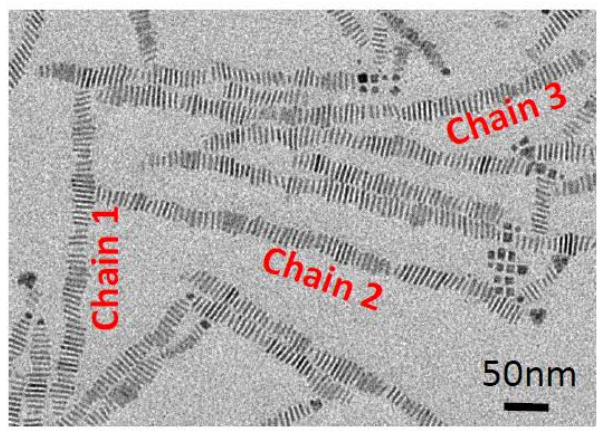

(c) Reference-5 $5^{\circ}$

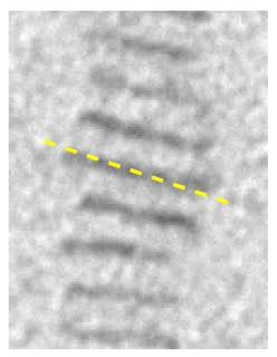

Reference- $3^{\circ}$

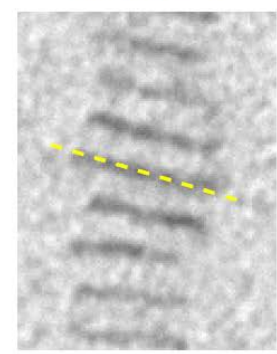

(b)

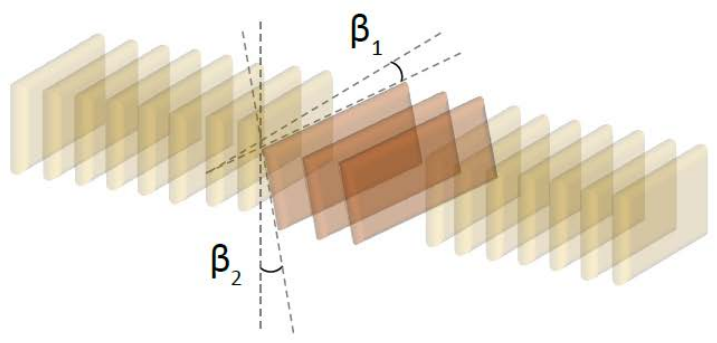

Reference

Reference $+3^{\circ}$

Reference $+5^{\circ}$
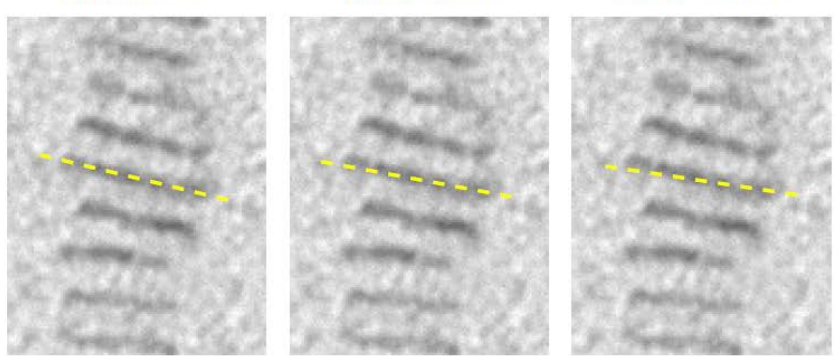

Figure S12 : (a) TEM image of various NPL chains. (b) Definition of tilt angles $\beta_{1}$ and $\beta_{2}$. (c) Estimation of the resolution on the tilt angle $\beta_{1}$. The yellow dashed line in the reference case (middle image) indicates the estimated orientation of the platelet. It shows clear deviations if we rotate the reference line by more than $\pm 3^{\circ}$.

The resulting distribution of angles is plotted on fig. S13 for three chains. For the first two ones, the mean of $\left|\beta_{1}\right|$ is $7^{\circ}$. For the third chain, the angles are more broadly distributed with a mean value of $\left|\beta_{1}\right|$ of $12^{\circ}$, however it may be explained by an overall bent shape of the chain (see fig. S12(a)), which is not representative of our optical measurements where we considered only chains deposited linearly. 


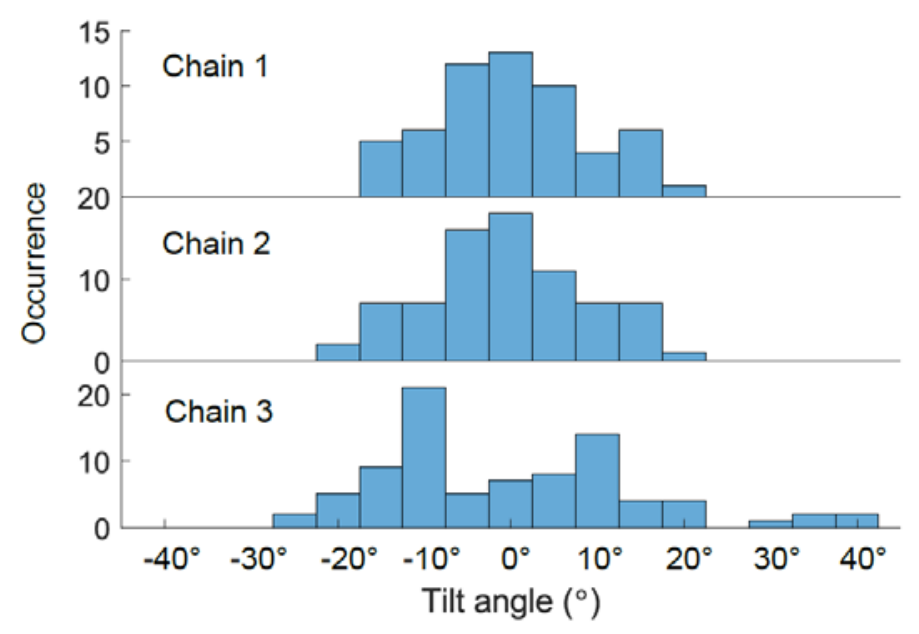

Figure S13 : Distribution of the tilt angle values $\beta_{1}$ measured for the NPLs of three NPL chains.

The effect of the tilt angle on the dipole contributions can be estimated by simple geometrical arguments. Let us first consider the in-plane angle $\beta_{1}$ (and keep $\beta_{2}=0$ ). If the platelet possesses two in-plane dipole components $\eta_{\|, 1}$ (horizontal) and $\eta_{\|, 2}$ (vertical) with no out-of-plane dipole $\left(\eta_{\perp}=0\right)$, the effect of the tilt is to create effective dipole contributions: $\eta_{\|, 1}^{\prime}=\cos ^{2} \beta_{1} \eta_{\|, 1}$ and $\eta_{\perp}^{\prime}=\sin ^{2} \beta_{1} \eta_{\|, 1}$ while the vertical component $\eta_{\|, 2}^{\prime}=\eta_{\|, 2}$ is not modified as it is not rotated. In order to account for an experimentally-measured ratio $\eta_{\perp}^{\prime} / \eta_{\|, 1}^{\prime}=0.12 / 0.62=0.19$, we would need a tilt angle $\tan ^{2} \beta_{1}=0.19$ so that $\beta_{1}=23^{\circ}$. On the other hand, if the assume that there is a tilt angle $\beta_{2}$ (while $\beta_{1}=0$ ), then $\eta_{\|, 2}^{\prime}=$ $\cos ^{2} \beta_{2} \eta_{\|, 2}$ and $\eta_{\perp}^{\prime}=\sin ^{2} \beta_{2} \eta_{\|, 2}$ so that an experimental ratio $\eta_{\perp}^{\prime} / \eta_{\|, 2}^{\prime}=0.12 / 0.25=$ 0.48 would be accounted for by a tilt angle $\beta_{2}=35^{\circ}$.

However, if we take into account the dielectric antenna effect, as explained in section C of this S.I. we would only need to account for a NPL of dipole parameters $\eta_{x}=0.08, \eta_{y}=$ $0.63, \eta_{z}=0.29$. This would require an in-plane tilt angle $\beta_{1}=20^{\circ}$, following the same calculations as above (now with $\eta_{\perp}^{\prime} / \eta_{\|, 1}^{\prime}=0.08 / 0.63=0.13$ ), or an out-of-plane angle $\beta_{2}$ $=28^{\circ}$. In either case, it would require angles significantly larger than the measured ones. If an angle $\beta_{1}=7^{\circ}$ is assumed, a ratio $\eta_{\perp}^{\prime} / \eta_{\|, 1}^{\prime}=0.015$ is found, leading to around $\eta_{\perp}=0.01$.

\section{$\mathrm{H}$ - Horizontal and vertical deposition of clusters}

Figure S14 shows additional TEM images of the batch of isolated (non assembled) platelets. Many platelets appear individually but some of them aggregate into clusters of more or less parallel stacked platelets. Some clusters deposit horizontally but it is then difficult to distinguish them from single platelets : they probably appear on fig. S14 as rectangles of darker shade. In some cases, the stacking is clearer because it is not well aligned : figure S14(b) shows some cases where the platelets overlap over only a portion of their surface. This diversity of cases may explain why the clusters show in fig. 5 a broader distribution of dipole coefficients. 
In a few cases (fig. S14(a)), the TEM images show clusters deposited vertically, on the edges of the platelets. It is then clear that the shape of each platelet is not planar and that there is a general twist of the cluster, in agreement with our discussion for the origin of the out-of-plane dipole.

(a)
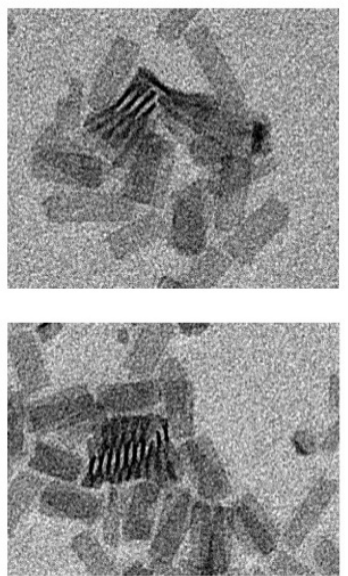

(b)
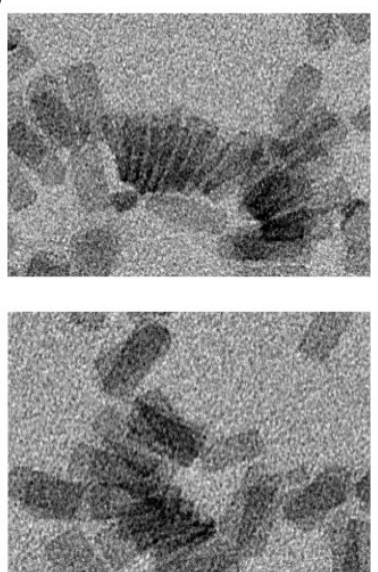

Figure S14 : TEM images of NPL clusters. (a) Examples of vertical clusters and small horizontal clusters. (b) examples of big misaligned horizontal clusters.

A few such cases of vertically-deposited clusters were found in the luminescence polarization and Fourier-plane analysis (fig. S15). For these emitters, the degree of polarization was significantly higher than for the horizontal clusters, and the vertical dipole contribution was much higher (here 0.33 ) while the second horizontal contribution was much weaker (here 0.09). For these reasons, these cases were attributed to vertically-deposited clusters, where the vertical contribution is the dipole along the NPL short axis, while the second horizontal contribution is the out-of-plane dipole. 
(a)

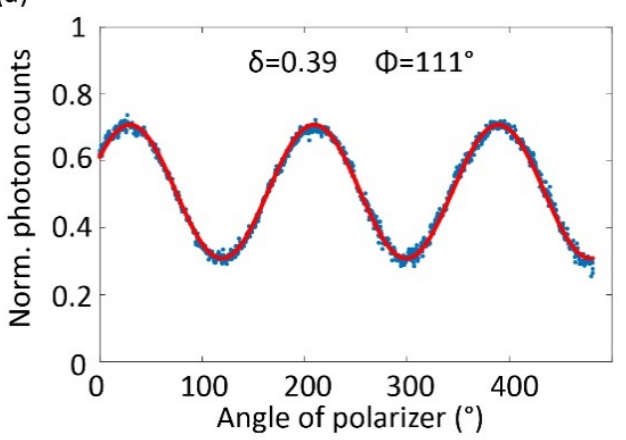

(c)

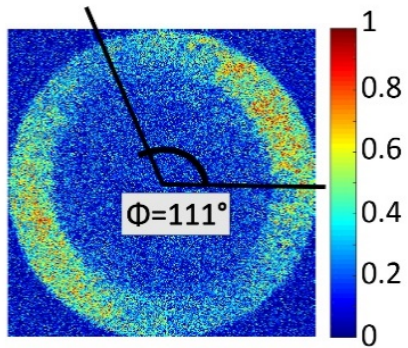

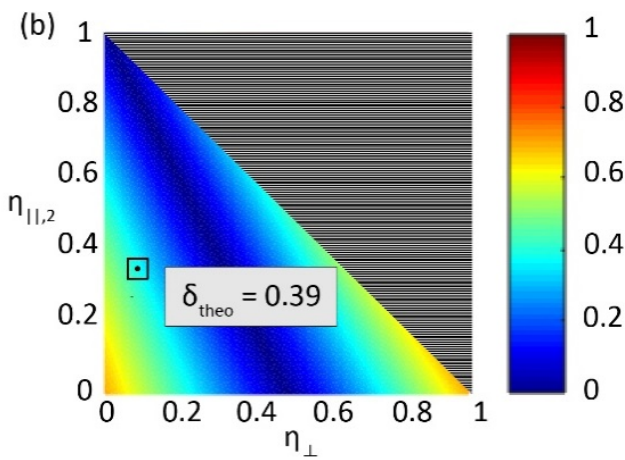

(d)

$$
\eta_{\perp}=0.09 \quad \eta_{\|, 2}=0.33
$$

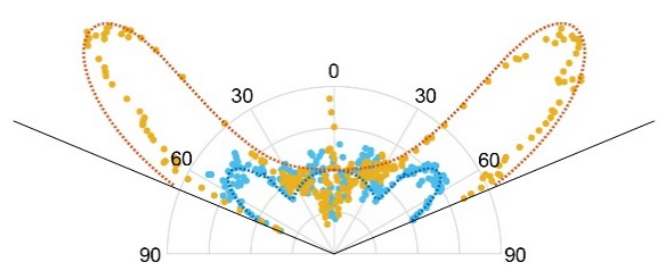

Figure S15 : (a) Polarimetric curve for a NPL cluster (on a glass substrate, covered by a 40-nm PMMA layer, observed with an oil immersion objective). (b) Theoretical degree of polarization (with the value 0.39 corresponding to the dipole coefficients extracted from the Fourier data). (c) Fourier imaging and (d) radiation pattern fitting for the same cluster.

$$
\eta_{\perp}=0.09 \quad \eta_{1,2}=0.33
$$

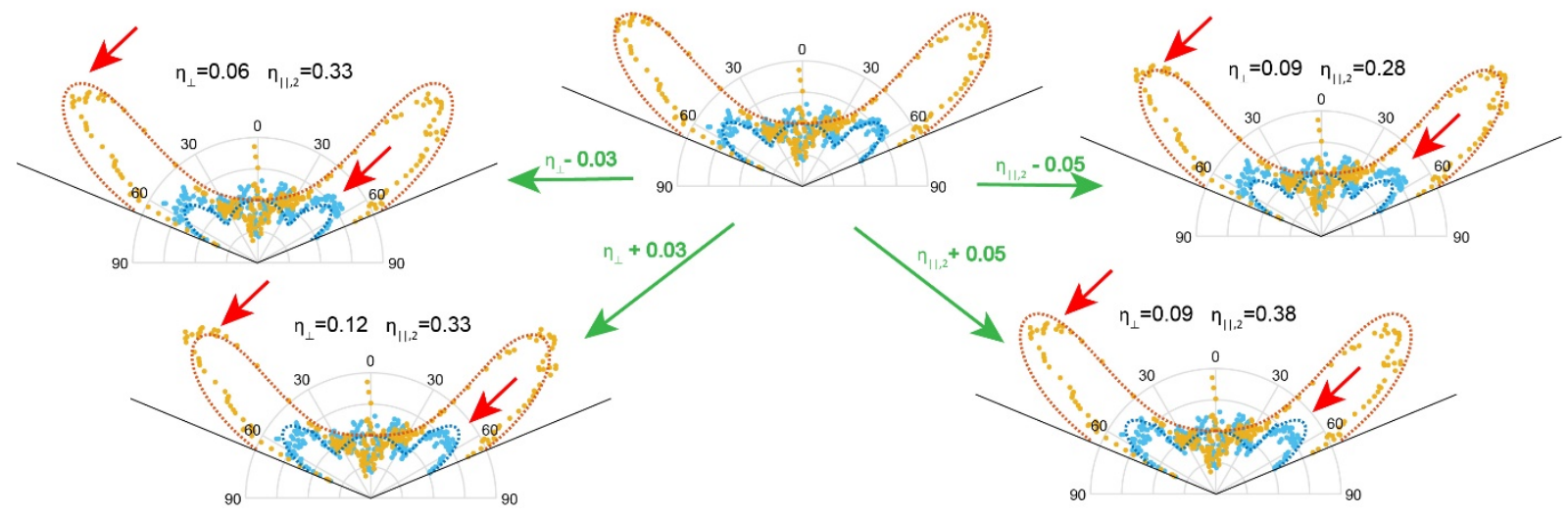

Figure S16 : Radiation pattern of fig. S15(d), fitted with different dipole coefficients.

We plot on figure S17 the measured out-of-plane dipole for the vertical clusters (green dots), along with the results for other emitters (as reported in fig. 6). The results for the vertical clusters are fully consistent with the others, with out-of-plane contributions of 0.09-0.11. 


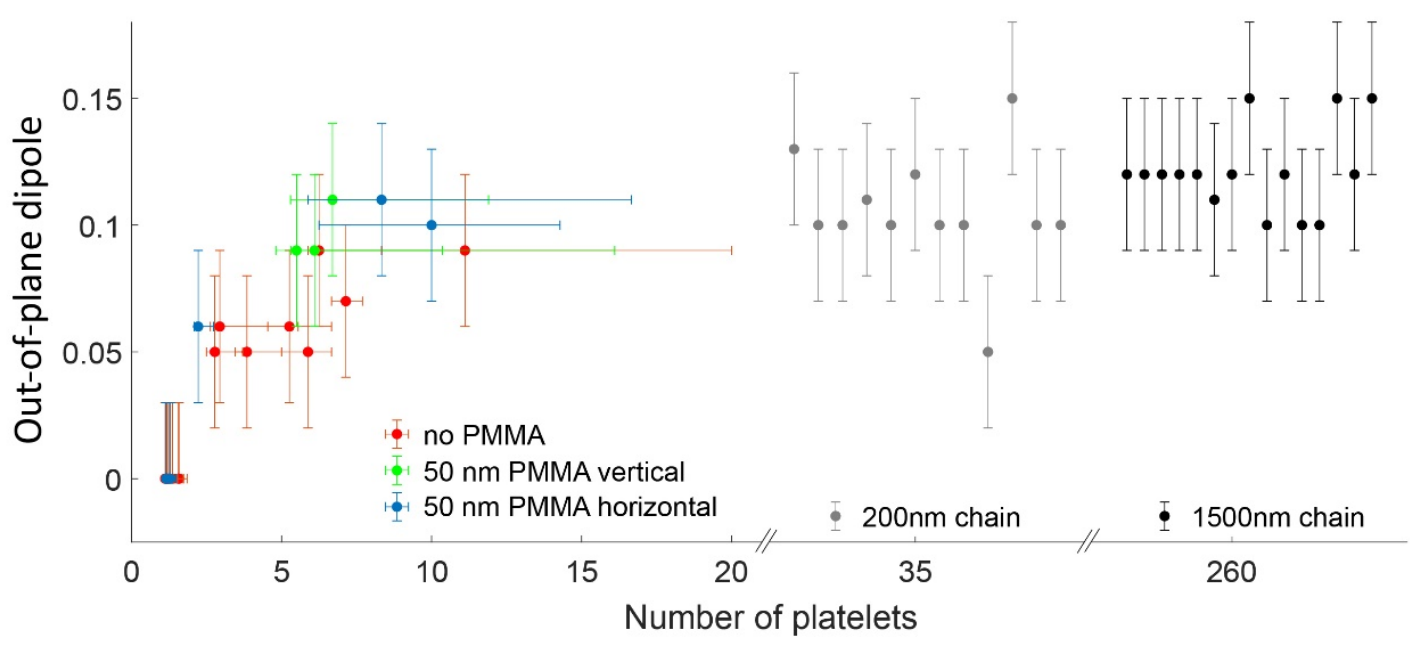

Figure S17 : summary of the measured out-of-plane dipoles as a function of the estimated number of platelets, like in figure 6, including the vertical clusters (in green).

\section{References}

[1] S. Ninomiya and S. Adachi, Optical properties of cubic and hexagonal CdSe, J. Appl. Phys. 78, 4681 (1995),

[2] J. A. Kurvits, M. Jiang and R. Zia, Comparative analysis of imaging configurations and objectives for Fourier microscopy, J. Opt. Soc. Am. A 32, 2082 (2015),

[3] B. Guzelturk, O. Erdem, M. Olutas, Y. Kelestemur and H. V. Demir, Stacking in colloidal nanoplatelets : tuning excitonic properties, ACS Nano 8, 12524 (2014),

[4] A. Antanovich, A. Prudnikau, A. Matsukovich, A. Achtstein and M. Artemyev, Self-assembly of CdSe nanoplatelets into stacks of controlled size induced by ligand exchange, J. Phys. Chem. C 120, 5764 (2016),

[5] Y. Gao, M. C. Weidman and W. A. Tisdale, CdSe nanoplatelet films with controlled orientation of their transition dipole moment, Nano Lett. 17, 3837 (2017),

[6] W. D. Kim, D.-E. Yoon, D. Kim, S. Koh, W. K. Bae, W.-S. Chae and D. C. Lee, Stacking of colloidal CdSe nanoplatelets into twisted ribbon superstructures, J. Phys. Chem C 123, 9445 (2019),

[7] J. Liu, L. Guillemeney, B. Abécassis and L. Coolen, Long range energy transfer in selfassembled stacks of semi-conducting nanoplatelets, Nano Lett. 20, 3465 (2020). 